\section{Effects of Physician Report Cards, Knowledge of Drug Prices, and Financial Incentives in Prescription Drug Costs}

Managed care pharmacists are often called upon by health plans, pharmacy benefit managers (PBMs), or medical groups to educate physicians about prescription drug prices and therapeutic alternatives. Managed care pharmacists who work in these roles find that physicians are not well informed about absolute and relative prescription drug prices. A survey of physicians in 2000 found that 20\% reported being "aware of actual drug costs" and 80\% reported lack of such knowledge; only 13\% reported receiving "formal education" about drug costs. ${ }^{1}$ Physicians were more sensitive to the cost of prescription drugs when patients had no health insurance. Physicians gave "strong consideration" to the cost of drugs when patients were self-paying (94\%) versus 68\% when patients had Medicare and 30\% when patients had either Medicaid or private health plan coverage of prescription drugs. Physicians' estimates of the cost of a month's supply of $33 \mathrm{com}-$ monly used drugs were accurate in $45 \%$ of cases, too low in $40 \%$ of cases, and too high in $15 \%$.

Evidence of the magnitude of the challenge in educating physicians about prescription drug prices can be found in the results of an educational intervention that involved (a) an interactive teaching conference and (b) distribution of a pocket guide, which listed the average wholesale prices of more than 100 medications commonly used in primary care. ${ }^{2}$ The study involved 146 internal medicine physicians in a before/after survey research design. Physicians after the intervention were more likely to ask patients about their out-of-pocket drug costs (22\% before versus $27 \%$ after, $P<0.01$ ) and less likely to feel unaware of drug costs ( $78 \%$ before versus $72 \%$ after, $P=0.02$ ). After the intervention, physicians also reported more concern about the cost of drugs when prescribing for patients with Medicare (58\% before versus $72 \%$ after, $P<0.01$ ) or no insurance $(90 \%$ before versus $98 \%$ after, $P<0.01)$. Knowledge of the costs of 33 drugs was more accurate after the intervention than before $(P<0.05)$. Yet, remarkably, after the intervention, $72 \%$ of physicians still felt "unaware of drug costs."

Physician profiling ("report cards") that includes information about the relative cost of therapeutic alternatives can affect average prescription drug costs. In a previous issue of the Journal, Yokoyama, Doan, Godley, et al. found that physician prescribing profiles combined with academic detailing that included relative price information for selective serotonin reuptake inhibitors (SSRIs) were associated with a reduction in the average cost per day of therapy of at least $11 \%$ over a 2 -year period. ${ }^{3}$ The combination of physician report cards and financial incentives can be influential in reducing prescription drug costs. A medical group in Michigan reported in 2003 that an incentive program that involved physician report cards for proton pump inhibitors (PPIs) and SSRIs was associated with a $6.8 \%$ reduction in prescription drug costs. ${ }^{4}$ Baseline data, differentiation of the effects of price versus utilization, and inflation-adjusted results were not presented, but the medical group reported that $73 \%$ of physicians who participated decreased their average costs in each of the 2 drug categories. The intervention involved prizes awarded to physicians who (a) recorded the lowest average drug cost for the 2 drug categories or (b) decreased their average overall drug cost by the largest percentage. The focus of the intervention was PPIs, SSRIs, overall generic drug use, and "judicious use of antibiotics."

\section{Clinical, Service, and Cost Outcomes} of Drug Coverage Edits and Quantity Limits

Like the lyrics of a popular song-"What have you done for me lately?"- drug plan sponsors and governmental agencies want to know what pharmacy benefit managers do to help manage care and cost outcomes. The penultimate goal of PBMs and managed care pharmacy is to demonstrate the value of interventions in the delivery, administration, and management of prescription drug and medical benefits. Two articles in this issue of the Journal measure the effects of pharmacy benefit management interventions on clinical, service-humanistic, or cost outcomes.

In a preliminary analysis to determine need for intervention, Hoffman, Mayzell, Pedan, Farrell, and Gilbert found that 24\% of health plan members who received a prescription for either a serotonin 5-HTl receptor agonist (e.g., sumatriptan [Imitrex]) or dihydroergotamine (DHE) nasal spray (i.e., Migranal) received a quantity sufficient in a 30-day period to exceed treatment of 4 headaches per month at the maximum daily dosage. ${ }^{5}$ The authors subsequently measured the utilization and cost outcomes of a program that imposed limits on coverage of migraine-abortive drugs, specifically, the triptans and DHE nasal spray. The authors found a $17 \%$ reduction in utilization of these target drugs and a 29\% reduction in direct drug costs of the target drugs. Presumably, the difference in reduced utilization versus cost was due, in part, to the use of prescriptions as the measure of utilization rather than days of therapy or units. ${ }^{6}$ The cost reduction is more remarkable since the authors used an inflation adjustment factor of only $4 \%$ per annum. The combined effects of drug price inflation and drug mix were about $10 \%$ to $11 \%$ per year during the 30 -month time period of this study, and drug utilization increased about $6 \%$ to $7 \%$ per year during this time..$^{7-9}$ In this context of rising drug "price," the combination of price increases and the mix of drugs, and rising drug utilization in general, the utilization and financial effects of the drug coverage limits studied by Hoffman, Mayzell, Pedan, Farrell, and Gilbert are more dramatic.

The extant study by Hoffman, Mayzell, Pedan, Farrell, and Gilbert did not measure clinical or service outcomes of the intervention, and it would have been valuable to know what happened to the nearly one quarter of triptan and DHE users who exceeded maximum dose guidelines. However, the effects on utilization and cost outcomes were significant. Overall costs of migraine-related drug therapy declined by $20 \%$, from $\$ 38.95$ per patient per month (PPPM) to $\$ 31.08$ PPPM. Total migrainerelated medical costs, also unadjusted for inflation, declined by $40 \%$, from $\$ 16.58$ PPPM to $\$ 9.94$, contributing to a reduction of $26 \%$ in the total (drug and medical) cost of migraine-related 